\title{
Research Article \\ Effects of Flow Compressibility on Two-Phase Mixing in Supersonic Droplet-Laden Flows
}

\author{
Zhaoxin Ren $\mathbb{D}^{1},{ }^{1}$ Bing Wang $\mathbb{D}^{2},{ }^{2}$ and Fan Zhang $\mathbb{D}^{3}$ \\ ${ }^{1}$ School of Power and Energy, Northwestern Polytechnical University, Xi'an 710072, China \\ ${ }^{2}$ School of Aerospace Engineering, Tsinghua University, Beijing 100084, China \\ ${ }^{3}$ China Academy of Launch Vehicle Technology, Beijing 100076, China
}

Correspondence should be addressed to Zhaoxin Ren; renzhaoxin@nwpu.edu.cn

Received 24 July 2020; Revised 13 October 2020; Accepted 11 November 2020; Published 8 December 2020

Academic Editor: Antonio Concilio

Copyright (c) 2020 Zhaoxin Ren et al. This is an open access article distributed under the Creative Commons Attribution License, which permits unrestricted use, distribution, and reproduction in any medium, provided the original work is properly cited.

\begin{abstract}
This research addresses a numerical analysis on the effects of flow compressibility on the characteristics of droplet dispersion, evaporation, and mixing of fuel and air according to the simulation of the spatially developing supersonic shear flows laden with evaporating $n$-decane droplets. A sixth-order hybrid WENO numerical scheme is employed for capturing the unsteady wave structures. The influence of inflow convective Mach number $\left(M_{c}\right)$, representing the high-speed flow compressibility, on the twophase mixing is analyzed, in which $M_{c}$ is specified from 0.4 to 1.0 . It is found that the shearing vortex is compressed spatially as $M_{c}$ increases, associated with the alternate distributions of compression and expansion regimes in the flow field. The flow compressibility changes not only the vortex structures but also the aerothermal parameters of the shear flows, and further influences the dispersion and evaporation of droplets. The two-phase mixing efficiency is observed to decrease as $M_{c}$ increases.
\end{abstract}

\section{Introduction}

Supersonic shear layer laden with dispersed fuel droplets is considered as the physical model for the supersonic c-based engine [1-4]. The research on supersonic two-phase flow includes the dispersion of sprayed droplet, the droplet evaporation, and the mixing between fuel vapors and air in the carrier phase, which are very important and complicated scientific issues for supersonic multiphase flow and combustion.

The unsteady entrainment due to the vortex dynamics promotes the fuel-oxidant mixing in supersonic shear flows. The velocity difference between the two parallel shearing flows results in the momentum transfer from the highspeed flow to the low-speed side, associated with the mass and heat transfer. The motion of vortices control the mixing process, and chemical reaction will not begin until the mixing reaches the molecular level. Therefore, the studies on the features of vortices with multiscales and their influence on combustion are of importance for engineering applications. Brown and Roshko [5] first found that the unsteady vortices have coherent structures when they experimentally studied the incompressible shear layers. The large-scale vortices formed in the shearing have a significant on the energy transport and mixing of species [6]. There are various sizes of flow structures in the shear layer. The Reynolds number, $\mathrm{Re}$, dominates the scale of the eddy structure. As Re increases, the small-scale vortex structures are found to be more and more abundant. For the compressible shear layers, the flow compressibility is of importance for the flow dynamics, and the convective Mach number, $M_{c}=\Delta U /\left(a_{1}+a_{2}\right)$, characterizes the flow compressibility (where $a_{1}$ and $a_{2}$ are the sound velocity of two streams). In addition, there are parameters, such as density ratio of two shear flows, gradient of velocity and pressure, and heat release, which are found to have an effect on the dynamics of the shear layer.

For the compressible shear layer, the variation of convective Mach number influences the development of the shear layer [7]. Clemens and Mungal [8] experimentally studied the compressible flow. They found that the shear flow develops more slowly with the increase of $M_{c}$, and the flow 
with higher speed has a more significant suppression. Kourta and Sauvage [9] and Vreman et al. [10] applied numerical simulations and indicated that the initiation of vortex shedding is depressed as the flow compressibility is intensified. In particular, as the $M_{c}$ rise above 1.2, the pairing process of vortices cannot be formed. Wang et al. [11] investigated the mixing process of supersonic shear layers by numerical simulations and focused on the influence of flow compressibility and gradients of velocity and density on the mixing efficiency. The results showed that the growth rates of shear layer and mixing efficiency are reduced with the increase of $M_{c}$. The increasing density ratio enlarges the shear layer thickness but has a negative effect on the mixing efficiency. The mixing efficiency is promoted but the shear layer thickness decreases with the increase of velocity ratio. In addition, the level of turbulence fluctuations represents the strength of turbulent eddies, and the previous researchers investigated the influence of flow compressibility on the turbulence fluctuations. Elliott and Samimy [12] applied the experimental studies on the compressible shear layer with High Re and showed that the fluctuating velocities and Reynolds stresses are reduced with the increase of $M_{c}$. Olsen and Dutton [13] used particle image velocimetry (PIV) for obtaining the velocity fields of the shear layer with weak compressibility. They found that the fluctuation of transverse velocity and Reynolds shear stress is reduced but the fluctuation of streamwise velocity remains constant, as compared with the incompressible flow. Pantano and Sarkar [14] numerically analyzed the turbulent shear layer with subsonic and supersonic speeds and found that the decrease of pressure-strain term contributes to the reduction of the turbulent fluctuation and the decreasing growth rate of the shear layer. Atoufi et al. [15] measured the compressible shear flow by large eddy simulations and studied the kinetic energy exchange. Their results showed that the production term is suppressed with the enhancement of flow compressibility, which results in the deceleration of the shear layer growth rate. The turbulent viscous dissipation term is also suppressed due to the increase of the flow compressibility.

The previous research provides a wealth of information on the characteristics of the shear layer thickness and the fluctuations of turbulence in the compressible shear layer flow. In general, the growth rate of the supersonic shear layer thickness is suppressed with the enhancement of the flow compressibility. The increase of $M_{c}$ leads to the suppression of pressure fluctuations, which prohibits the momentum transfer between the vortices, associated with the decrease of entrainment rates. In addition, the reduction of the production terms from the turbulent kinetic energy correlates with the suppression of the turbulent diffusion. However, it is noteworthy that the studies are absent for the influence of the flow compressibility on the droplet evaporation and two-phase mixing in supersonic shear flows despite its importance for understating the physics of the spray combustion in the supersonic flow. The previous research has shown that the high-speed flow compressibility affects the flow dynamics, which can expect to influence the dispersion and evaporation of droplets and fuel-air mixing. Furthermore, the ignition and flame will be altered in the supersonic combustor of the scramjet. The related physics should be revealed.
The present study is aimed at evaluating the impact of flow compressibility on the two-phase mixing in a supersonic shear layer laden with fuel $\left(n\right.$-decane, $\left.\mathrm{C}_{10} \mathrm{H}_{22}\right)$ droplets. The numerical simulations with high order schemes are applied to mimic the unsteady two-phase flow. The convective Mach number $\left(M_{c}\right)$ increases from 0.4 to 1.0. The remainder of this paper is organized as follows. The governing equations, numerical methods, and validations are introduced in Section 2. The results are presented in Section 3, including the effects of flow compressibility on the carrier flow, and the features of droplet dispersion, evaporation, and fuel-air mixing. Finally, the concluding remark and discussion are drawn in Section 4.

\section{Governing Equations and Numerical Methods}

2.1. Governing Equations for Fluid Phase. In this paper, the motion of inertial evaporating fuel droplets is considered in the compressible shear layer. The governing equations, comprising the equations of mass, momentum, and energy and transport equations of three chemical species $\left(\mathrm{C}_{10} \mathrm{H}_{22}\right.$, $\mathrm{O}_{2}, \mathrm{~N}_{2}$ ), supplemented by the equation of state for the ideal multispecies gas, are solved in the following form:

$$
\begin{gathered}
\frac{\partial \rho}{\partial t}+\frac{\partial}{\partial x_{j}}\left(\rho u_{j}\right)=S_{\mathrm{m}} \\
\frac{\partial}{\partial t}\left(\rho u_{i}\right)+\frac{\partial}{\partial x_{j}}\left(\rho u_{i} u_{j}+P \delta_{i j}-\tau_{i j}\right)=S_{\mathrm{F}, i} \\
\frac{\partial}{\partial t}\left(\rho e_{\mathrm{t}}\right)+\frac{\partial}{\partial x_{j}}\left(\left(\rho e_{\mathrm{t}}+P\right) u_{j}-u_{i} \tau_{i j}-q_{j}\right)=S_{\mathrm{Q}} \\
\frac{\partial}{\partial t}\left(\rho Y_{k}\right)+\frac{\partial}{\partial x_{j}}\left(\rho Y_{k} u_{j}\right)+\frac{\partial}{\partial x_{j}}\left(\rho Y_{k}\left(V_{k, j}+V_{j}^{\mathrm{c}}\right)\right)=S_{Y_{k}} \\
P=\rho R T \sum_{k=1}^{N_{S}} \frac{Y_{k}}{W_{k}}
\end{gathered}
$$

where $\rho$ is the density, $u_{i}$ is the velocity in the $i$ th direction, $P$ is the static pressure, and $T$ is the temperature. $R$ is the universal gas constant. $e_{t}$ is the total energy (kinetic energy plus internal energy) and is defined as

$$
e_{\mathrm{t}}=\sum_{k=1}^{N_{S}} Y_{k}\left(\int_{T_{\mathrm{ref}}}^{T} c_{p, k} \mathrm{~d} T+h_{f, k}^{0}\right)-\frac{P}{\rho}+\frac{u_{i} u_{i}}{2}
$$

$c_{p, k}$ is the specific heat capacity at constant pressure, and $h_{f, k}^{0}$ is the specific chemical formation enthalpy at the reference temperature, $T_{\text {ref }}$. The values of $c_{p, k}$ are calculated by a fifth-order polynomial [16]. $\tau_{i j}$ is the Newtonian viscous stress tensor, and $\mu$ is the viscosity. $Y_{k}$ is the mass fraction of the $k^{\text {th }}$ species. $V c j$ is the correction velocity. $N_{S}$ is the total number of the chemical species, and $W_{k}$ is the molecule weight of the $k^{\text {th }}$ species. For the transport properties, the 
kinetic theory [17] for a gaseous mixture is utilized. In particular, the Lennard-Jones potentials are used to obtain the intermolecular forces. The heat conductivity of each species is calculated by using the modified Eucken model. The dynamic viscosity and the binary diffusion coefficient are from the Chapman-Enskog theory.

$$
\begin{aligned}
\mu_{k} & =\frac{26.69 \sqrt{W_{k} T}}{\sigma_{k}^{2} \Omega_{v, k}}, \\
\frac{\lambda_{k} W_{k}}{\mu_{k} C_{v, k}} & =1.32+\frac{1.77}{\left(C_{p . k} / R\right)-1}, \\
D_{\mathrm{AB}} & =\frac{0.00266 T^{3 / 2}}{P W_{A B}^{1 / 2} \sigma_{A B}^{2} \Omega_{D}} .
\end{aligned}
$$

Here, $\sigma_{k}$ is the hard-sphere diameter of the $k^{\text {th }}$ species. $D_{\mathrm{AB}}$ is the diffusion coefficients for the binary gas $A$ and $B$, $W_{\mathrm{AB}}$ is the combined molecular weights of $A$ and $B . \sigma_{\mathrm{AB}}$ is the characteristic length of the intermolecular force law, and $\Omega_{D}$ is the collision integral for diffusion. The semiempirical expressions proposed by Wake and Wassiljewa are used to calculate the dynamic viscosity, $\mu$, and heat conductivity, $\lambda$, of the gaseous mixture. The right-hand side terms $S_{\mathrm{m}}$, $S_{\mathrm{F}, i}$, and $S_{\mathrm{Q}}$ describing the interphase couplings of mass, momentum, and energy, respectively, and they are calculated via summating the total number of droplets $N$, existing in the grid, $\Delta V$, of the gas phase calculation,

$$
\begin{aligned}
& S_{m}=-\frac{1}{\Delta V} \sum_{N}\left(\dot{m}_{\mathrm{d}}\right), \\
& S_{\mathrm{F}, i}=-\frac{1}{\Delta V} \sum_{N}\left(F_{\mathrm{d}, i}+\dot{m}_{\mathrm{d}} u_{\mathrm{d}, i}\right), \\
& S_{\mathrm{Q}}=-\frac{1}{\Delta V} \sum_{N}\left(Q_{\mathrm{d}}+\dot{m}_{\mathrm{d}}\left(\frac{u_{\mathrm{d}, i} u_{\mathrm{d}, i}}{2}+h_{\mathrm{V}, \mathrm{ff}}\right)\right) \text {, } \\
& S_{Y_{k}}= \begin{cases}-\frac{1}{\Delta V} \sum_{N}\left(\dot{m}_{\mathrm{d}}\right) & \text { for fuel, } \\
0 & \text { for other species. }\end{cases}
\end{aligned}
$$

Here, $m_{d}$ and $\dot{m}_{d}$ are the droplet mass and the mass change rate, respectively. $u_{\mathrm{d}, i}$ is the droplet velocity. $h_{\mathrm{V}, \mathrm{sf}}$ is the evaporated vapor enthalpy at the droplet surface.

2.2. Governing Equations for Droplet Phase. The dispersion of individual droplet in the supersonic flow is tracked by using the Lagrangian trajectory model. The droplets are considered as sparsely dispersion, and the droplets do not influence each other. The drag force due to the interphase slip velocity acting on the droplet is modeled, and the heat convection is calculated. Under the assumption made as such, the position $\left(x_{\mathrm{d}, i}\right)$, velocity $\left(u_{\mathrm{d}, i}\right)$, temperature $\left(T_{d}\right)$, and mass $\left(m_{d}\right)$ of a single droplet are controlled by the following equations [18]:

$$
\frac{d x_{d, i}}{d t}=u_{d, i}
$$

$$
\begin{gathered}
\frac{d u_{d, i}}{d t}=\frac{F_{d}}{m_{d}}=\left(\frac{f_{F}\left(\operatorname{Re}_{d}\right)}{\tau_{d}}\right)\left(u_{i @ d}-u_{d, i}\right), \\
\frac{d T_{d}}{d t}=\frac{Q_{d}+\dot{m}_{d} L_{V}}{m_{d} c_{L}}=\left(\frac{f_{Q}}{\tau_{d}}\right)\left(\frac{\mathrm{Nu}}{3 \operatorname{Pr}}\right)\left(\frac{c_{p}}{c_{L}}\right)\left(T_{@ d}-T_{d}\right)+\left(\frac{\dot{m}_{d}}{m_{d}}\right) \frac{L_{V}}{c_{L}}, \\
\frac{d m_{d}}{d t}=\dot{m}_{d}=-m_{d}\left(\frac{1}{\tau_{d}}\right)\left(\frac{\mathrm{Sh}}{3 \mathrm{Sc}}\right) \operatorname{In}\left(1+B_{M}\right),
\end{gathered}
$$

where $u_{i @ d}$ and $T_{@ \mathrm{~d}}$ are the velocity and temperature of carrier flow at the droplet position. $c_{p}$ is the specific heat of gas surrounding the droplet, $c_{L}$ is the specific heat of the droplet, and $L_{V}$ is the latent heat from evaporation. The momentum response time, $\tau_{d}$, is as follows,

$$
\tau_{\mathrm{d}}=\frac{\rho_{\mathrm{d}} d_{\mathrm{d}}^{2}}{18 \mu}
$$

where $d_{\mathrm{d}}$ is the droplet diameter. For the drag force, the correction function, $f_{F}\left(\operatorname{Re}_{d}\right)$, for the influence of droplet Reynolds number, $\operatorname{Re}_{d}$, is

$f_{F}\left(\mathrm{Re}_{d}\right)=\frac{\mathrm{Re}_{d}}{24}\left(\frac{24}{\mathrm{Re}_{\mathrm{d}}}\left(1+0.15 \mathrm{Re}_{d}^{0.687}\right)+\frac{0.42}{1+42500 \mathrm{Re}_{d}^{-1.16}}\right)$ for $\mathrm{Re}_{d}<2 \times 10^{5}$.

$\operatorname{Re}_{d}=\left|u_{i}-u_{d, i}\right| d_{d} / v$ is based on the slip velocity between the droplet and the local gas. $\operatorname{Pr}=\mu c_{p} / \lambda$ and $\mathrm{Sc}=\mu / \rho D$ are Prandtl and Schmidt numbers, respectively. The Nusselt and Sherwood numbers are $\mathrm{Nu}=2+0.552 \operatorname{Re}_{d}^{1 / 2} \operatorname{Pr}^{1 / 3}$ and $\mathrm{Sh}=2+0.552 \mathrm{Re}_{d}^{1 / 2} \mathrm{Sc}^{1 / 3}$, respectively. The evaporation rate of droplets is calculated according to the mass transfer number, $B_{M}=\left(Y_{\mathrm{sf}}-Y_{V}\right) /\left(1-Y_{\mathrm{sf}}\right) . Y_{V}$ is the fuel mass fraction on the far-field, and $Y_{\text {sf }}$ is the fuel mass fraction at the droplet interface, which is obtained directly from the surface molar fraction $\left(\chi_{\mathrm{sf}}\right)$ based on a nonequilibrium evaporation model [19],

$$
\begin{gathered}
Y_{\mathrm{sf}}=\frac{\chi_{\mathrm{sf}}}{\chi_{\mathrm{sf}}+\left(1-\chi_{\mathrm{sf}}\right) W / W_{V}}, \\
\chi_{\mathrm{sf}}=\frac{P_{\mathrm{atm}}}{P} \exp \left(\frac{L_{V}}{R / W_{V}}\left(\frac{1}{T_{B, L}}-\frac{1}{T_{d}}\right)\right)-\frac{2 L_{k}}{d_{d}} \beta, \\
L_{k}=\frac{\mu\left(2 \pi T_{\mathrm{d}}\left(R / W_{V}\right)\right)^{1 / 2}}{\mathrm{Sc} P} .
\end{gathered}
$$

$W$ is the molecular weight of the carrier gas, $W_{V}$ is the molecular weight of the fuel vapor, $P_{\text {atm }}$ is the atmospheric pressure, and $T_{B, \mathrm{~L}}$ is the liquid boiling temperature at $P_{\mathrm{atm}}$. $L_{k}$ is the Knudsen layer thickness.

2.3. Numerical Methods. The supersonic shear flow laden with droplets is simulated by our in-house code, which has been utilized for the studies of supersonic flow and twophase reacting flow $[18,20]$. The numerical methods are summarized below. A finite difference methodology is performed. The convection fluxes are calculated by an 


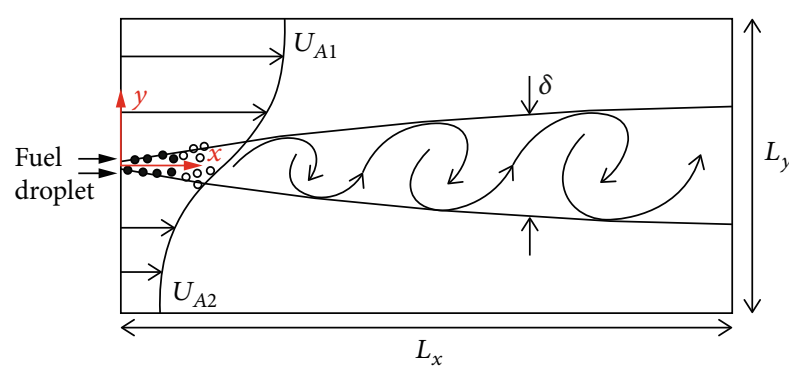

FIGURE 1: Schematic of the supersonic shear layer laden with droplets released from the inlet center.

adaptive central-upwind sixth-order WENO (WENO-CU6) scheme [21] for obtaining the smooth turbulent fields and for capturing the eddy shocklets and complex waves in the supersonic flow with strong compressibility. The viscous terms are discretized according to a sixth-order symmetrical compact difference scheme. For the time advancement of the governing equations for the gas phase, a third-order explicit Runge-Kutta method is applied. A third-order Adams approach is used for the time advancement for the droplet equations. In order to obtain the velocity, temperature, and other properties of the flow around the droplet, a fourthorder Lagrangian interpolation method is utilized. The dispersed droplets are treated as point sources, and the influence of the droplets on the carrier flow is modeled by adding the source terms, $S$, to the grids around the droplets, namely, the particle-source-in cell (PSI-CELL) model.

2.4. Numerical Setup and Simulation Parameters. The schematic diagram of the present computational configuration is depicted in Figure 1. As shown, the shear layer is formed by the upper and lower streams moving in a same direction with different speeds $U_{A 1}$ and $U_{A 2}$. The letters $x$ and $y$ refer to the streamwise and transverse directions, respectively. The streamwise and transverse domain lengths are $L_{x}$ and $L_{y}$, respectively. $L_{x}$ is set to $3000 \delta_{0}$ and $L_{y}$ is set to $750 \delta_{0}$. The initial mixing layer thickness is $\delta_{0}$. By setting $L_{x}=4 L_{y}$, the transverse domain size is large enough to have minimal influence on the main interaction region of the dropletladen shear layer. Stream 1 and 2 are both air $(T=700 \mathrm{~K}, P$ $=0.1 \mathrm{MPa})$ as a mixture of nitrogen $\left(\mathrm{N}_{2}\right)$ and oxygen $\left(\mathrm{O}_{2}\right)$ with (in terms of mass fraction) $Y_{\mathrm{N}_{2}}=0.77$ and $Y_{\mathrm{O}_{2}}=0.23$. The velocity ratio of two flows, $U_{A 2} / U_{A 1}$, equals to 0.6 . At the inflow boundary, the streamwise velocity distribution is specified as a hyperbolic tangent profile by using a supersonic inlet boundary condition as follows,

$$
\begin{gathered}
u(x=0, y)=\frac{U_{1}+U_{2}}{2}+\frac{U_{1}-U_{2}}{2} \tanh \left(\frac{y}{2 \delta_{0}}\right), \\
v(x=0, y)=0 .
\end{gathered}
$$

The transverse perturbations are added to the inlet velocity in order to excite the growth of spanwise disturbances, and the random perturbations are added to the transverse component of the inflow velocity based on the most-unstable frequency in the flow field [22],
TABLE 1: Simulation cases.

\begin{tabular}{lcc}
\hline Case & Mach number, $M_{A 1}, M_{A 2}$ & Convective Mach number, $M_{c}$ \\
\hline $0.4 M_{c}$ & $2.0,1.2$ & 0.4 \\
$0.6 M_{c}$ & $3.0,1.8$ & 0.6 \\
$0.8 M_{c}$ & $4.0,2.4$ & 0.8 \\
$1.0 M_{c}$ & $5.0,3.0$ & 1.0 \\
\hline
\end{tabular}

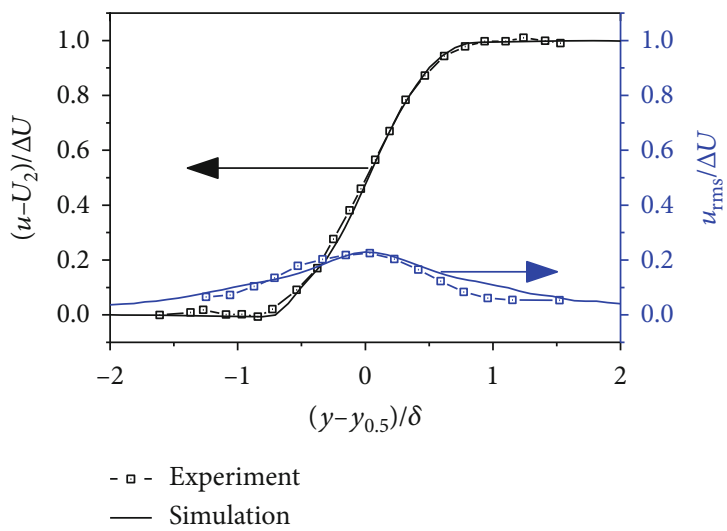

FIGURE 2: Comparison of the simulation results with experiment data for a supersonic shear layer.

$$
\begin{gathered}
v^{\prime}(x=0, y)=\left(U_{1}-U_{2}\right) G(y) A \sin (2 \pi f t+\xi), \\
u^{\prime}(x=0, y)=0,
\end{gathered}
$$

where $G(y)$ is the Gaussian function, $A$ is the amplitude, and $f$ is the most unstable frequency. The random phase is $\xi$. The most unstable frequency is usually obtained by means of the flow stability analysis based on the base flow profile given by Eq. [15]. The nonreflecting boundary conditions are set in the transverse directions [23]. At the outlet, the boundary conditions are zero-gradients that are interpolated by assuming first-order derivatives of all flow parameters.

Pure $n$-decane spray is injected at the centerline with a width of $5 \delta_{0}$. The droplets are initially randomly seeded, with the same size. The initial droplet velocity is identical to the local gas velocity, and the initial droplet temperature is $T_{d}$ $=298.15 \mathrm{~K}$. The liquid fuel is preatomized, and the droplets are considered without further breakup process, since the Weber number based on the slip velocity (the initial velocity between the two phases is zero) is quite small for the concerned droplets in this study.

Table 1 provides the inlet parameters for the present simulation cases. Since the velocity ratio, $U_{A 2} / U_{A 1}$, has an important effect on the development of the shear layer flow, the velocity ratio is the same for all the simulation cases and the inflow Mach numbers of two streams; $M_{A 1}$ and $M_{A 2}$ are changed to study the influence of the flow compressibility and to exclude the interference of other factors. The convection Mach number, $M_{c}$, increases from 0.4 to 1.0 in the four simulation cases, associated with the strength of flow 

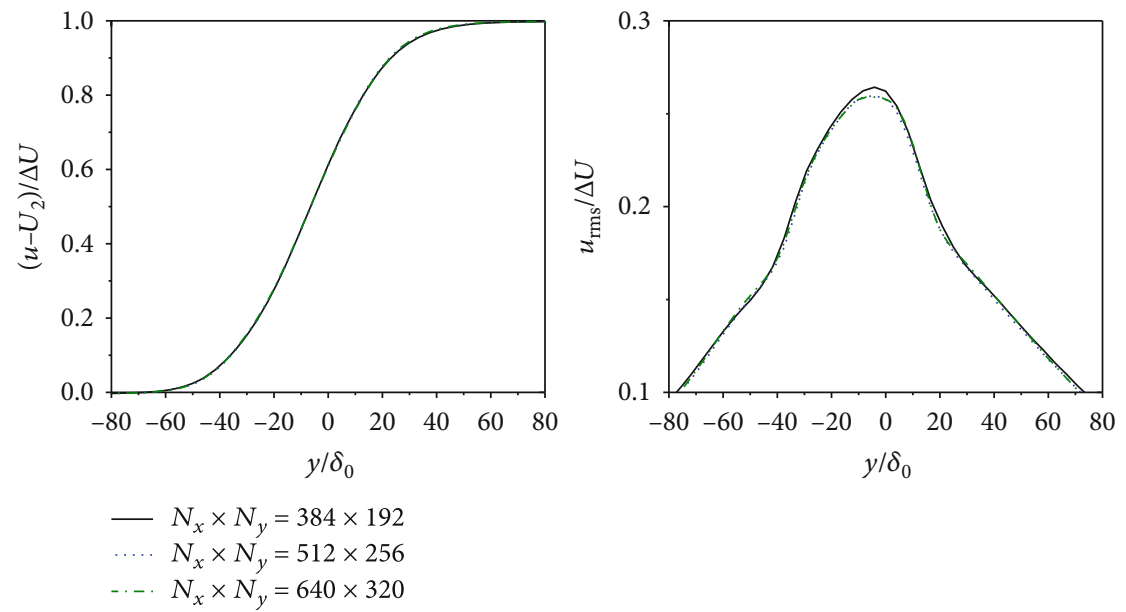

$\cdots N_{x} \times N_{y}=512 \times 256$

‥ $N_{x} \times N_{y}=640 \times 320$

FIgURe 3: Comparison of numerical results at different grid sets: (left) mean streamwise velocity and (right) r.m.s streamwise velocity.
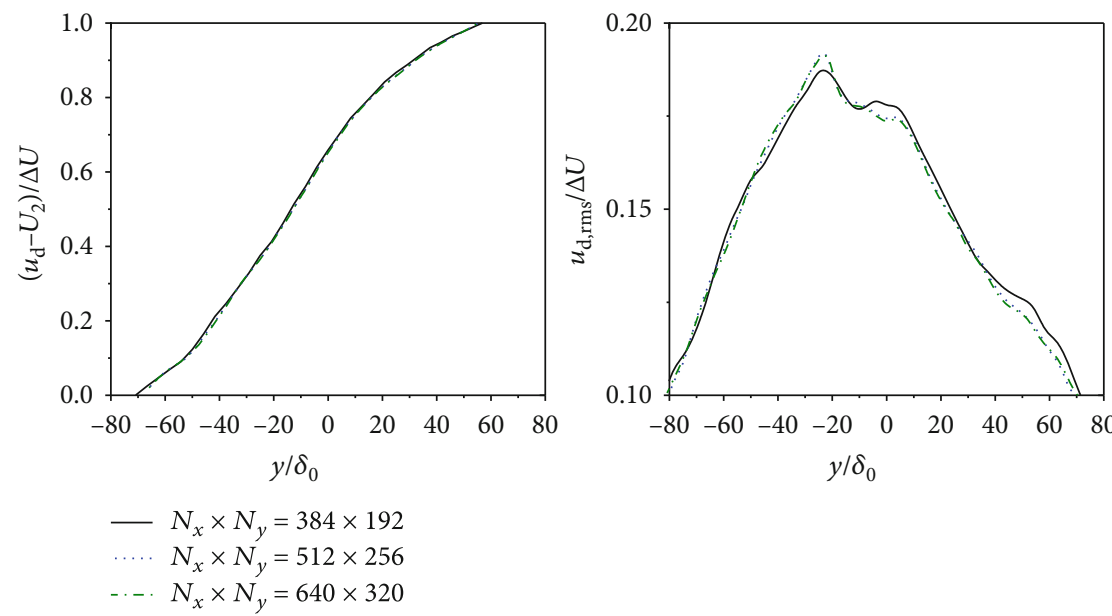

$\begin{aligned} N_{x} \times N_{y} & =512 \times 256 \\ N_{x} \times N_{y} & =640 \times 320\end{aligned}$

Figure 4: Comparison of numerical results at different grid sets: (left) mean streamwise velocity and (right) r.m.s streamwise velocity of droplets.

compressibility. Generally, the shear flow is considered to have strong compressibility with compression waves or shocklets, and the corresponding convection Mach number is $M_{c}>0.8$. The present flows in this study consider both weak and strong flow compressibility. The cold droplet is injected into the hot stream to evaporate. The spray equivalence ratio, $\Phi_{0}$, is defined as $\Phi_{0} \cdot(\mathrm{F} / \mathrm{O})_{\text {st }}=\dot{m}_{\text {spray }} /\left(\dot{m}_{\text {air }} Y_{\mathrm{O}_{2}}\right)$. Here, $(\mathrm{F} / \mathrm{O})_{\text {st }}$ is the stoichiometric fuel-to-oxidizer ratio. $\dot{m}_{\text {spray }}$ and $\dot{m}_{\text {air }}$ are the mass flow rates of fuel and air, respectively. The high $\Phi_{0}$ means that more fuel enters the flow field, which causes the influence of the dispersed phase on the local carrier flow (turbulence modulation). In the present study, the effects of flow compressibility are considered, and a low $\Phi_{0}\left(\Phi_{0}=0.1\right)$ is selected for all simulation cases. The inflow velocities in these cases are different, and if the initial diameter of the droplets in each case is the same, the residence time of the droplets in the flow is expected to be different. In order to ensure the same residence time of the droplets in different cases, the Vaporization Damköhler number [24], $\mathrm{Da}_{\text {vapor }}$, in the four cases keeps the same, and the expression of $\mathrm{Da}_{\text {vapor }}$ is as follows,

$$
\mathrm{Da}_{\text {vapor }}=\frac{t_{r}}{t_{v}}=\frac{L_{\mathrm{P}} / \bar{U}_{A}}{\left(\rho_{d} d_{d}^{2} c_{p}\right) / 12 \lambda} \propto \frac{1}{\bar{U}_{A} d_{d, 0}^{2}} .
$$

Here $t_{r}$ is the residence time of the droplets in the supersonic flow, which is estimated as the ratio of the living distance $L_{P}$ of the droplets to the averaged inflow velocity, and $t_{v}$ is the evaporation time of the droplets [25]. Although the residence time and the evaporation time of the droplets are different in each case, the $\mathrm{Da}_{\text {vapor }}$ is the same and hence the simulation cases become comparable. Therefore, the influence of flow compressibility on the droplet phase can be studied.

2.5. Model Validation. Here, the computation of a supersonic shear layer without droplets is performed for the validation of the ability to simulate the gaseous flow. The experiment data for the supersonic shear layer conducted by Goebel et al. [26] is used for the comparison. The self-similarity profiles of the mean streamwise velocity and the standard deviation of the streamwise velocity are found to be same with the experimental measurements, as shown in Figure 2. 


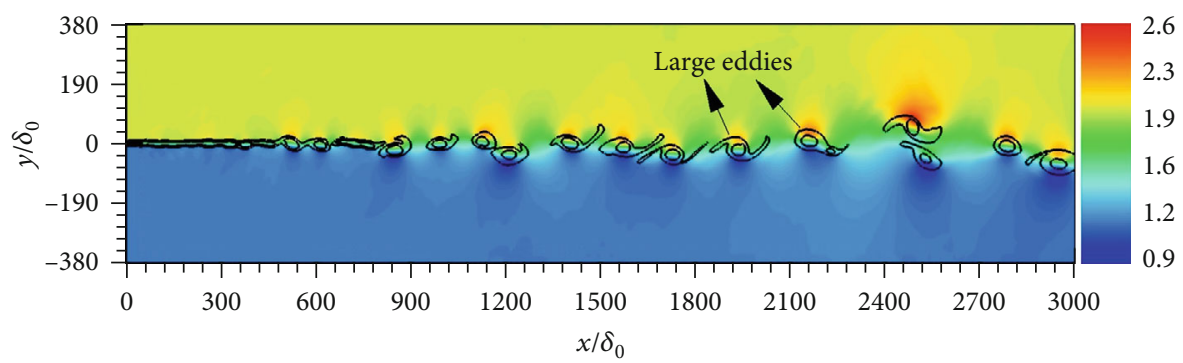

(a)

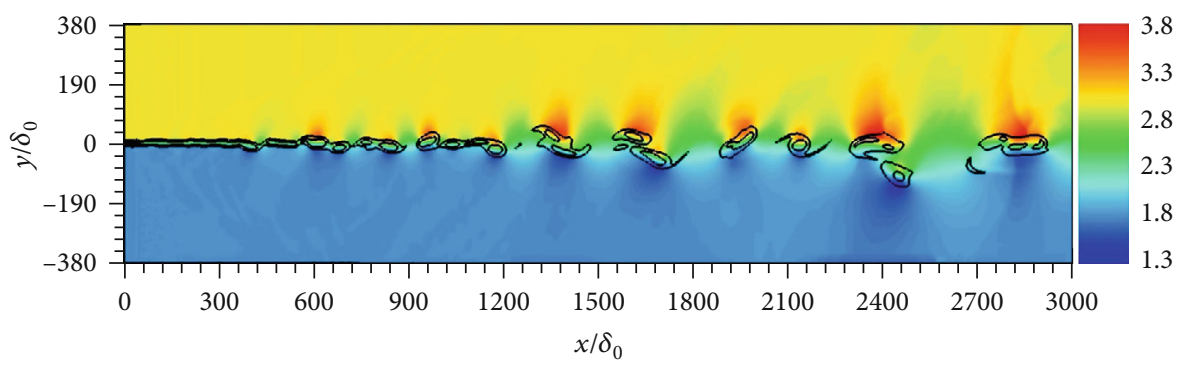

(b)

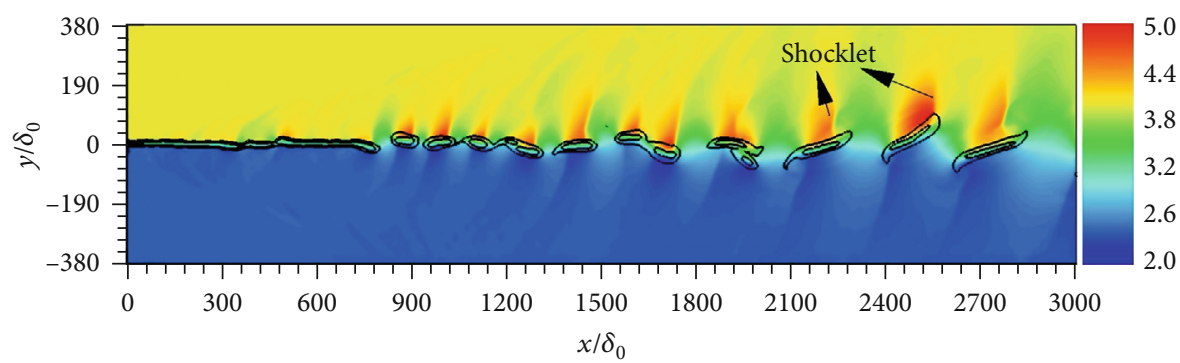

(c)

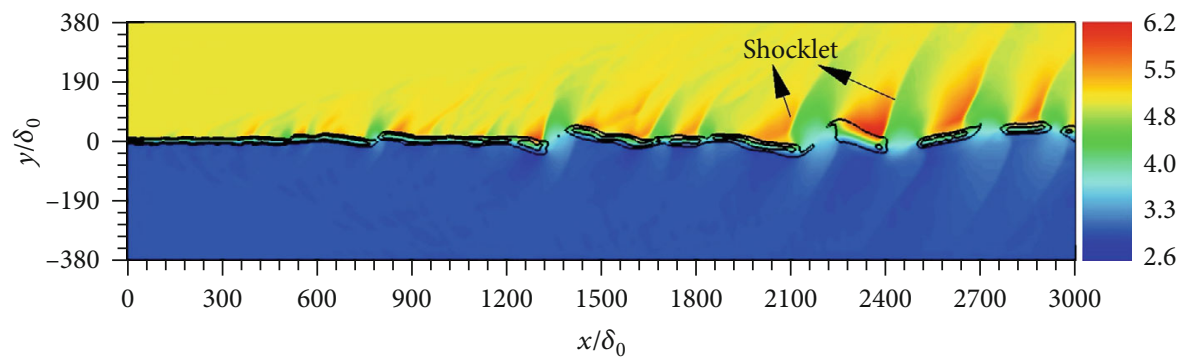

(d)

FIGURE 5: Instantaneous distributions of Mach numbers: (a) $0.4 M_{c}$, (b) $0.6 M_{c}$, (c) $0.8 M_{c}$, and (d) $1.0 M_{c}$. Here, the black lines refer to dimensionless spanwise vorticity $\left(\Omega_{\mathrm{z}} /\left(\Delta U_{\mathrm{A}} / \delta_{0}\right)=[-0.02,-0.005]\right.$.

This validation verifies that the present code has the ability to mimic the supersonic shear flow.

For the droplet evaporation in the sparse spray, the evaporation of single droplet is slightly affected by the surrounding droplets. The numerical simulation is applied for the experiments of a single droplet evaporation [27]. The comparisons between the numerical simulations and experiments show that the nonequilibrium evaporation model used in the present study can reproduce the temporal evolution of the squared droplet diameter [18].

The grid scale should be carefully designed, and the study of grid independence is applied. Three sizes of grids are utilized,

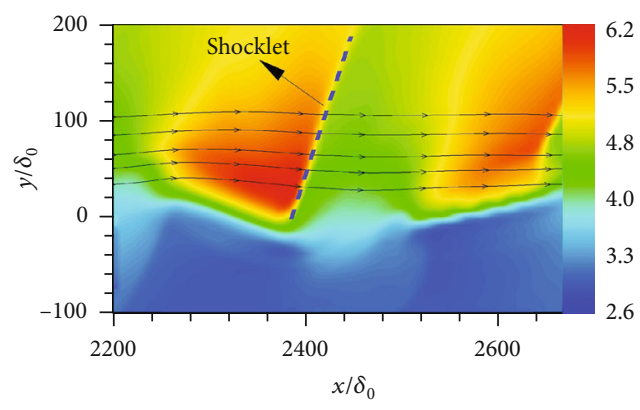

FIgURE 6: Local enlargement of the shocklet in Figure 2(d) with $M_{c}=1.0$. 


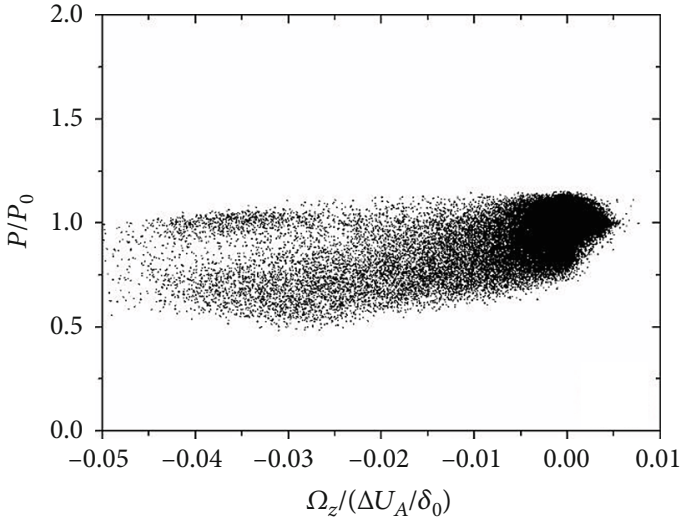

(a)

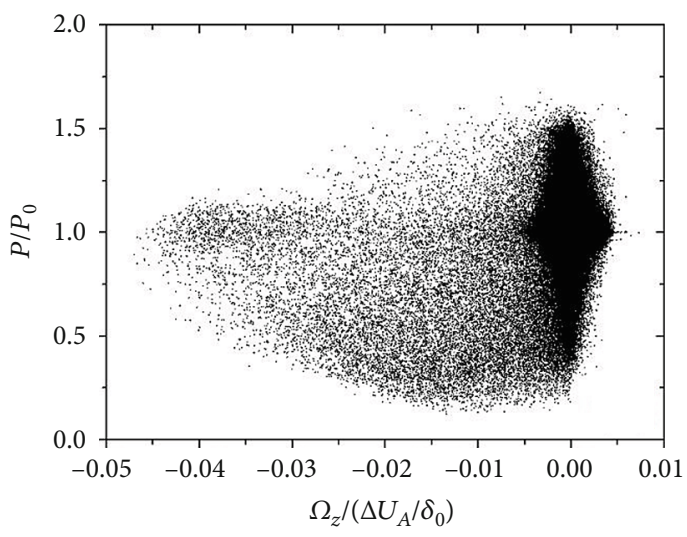

(c)

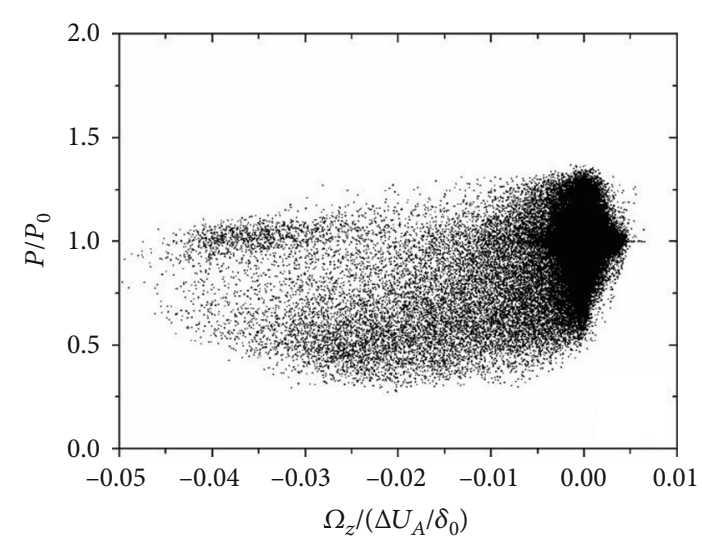

(b)

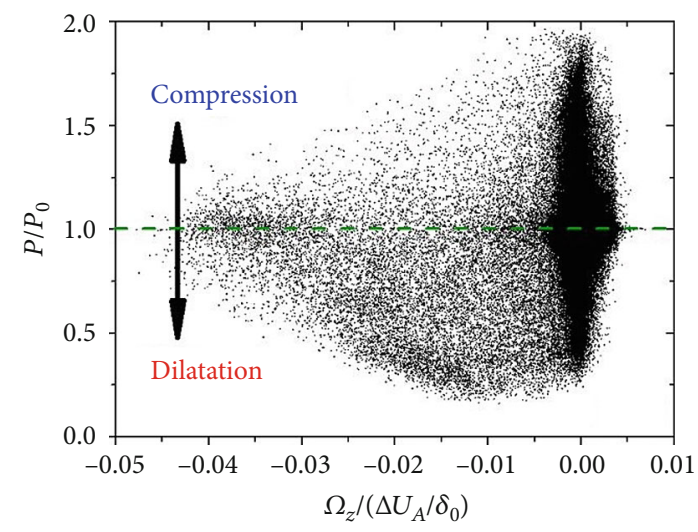

(d)

Figure 7: Scatter distributions of dimensionless pressure, $P / P_{0}$, in the coordinates of dimensionless spanwise vorticity, $\Omega_{\mathrm{z}} /\left(\Delta U_{\mathrm{A}} / \delta_{0}\right)$ : $($ a) 0.4 $M_{c}$, (b) $0.6 M_{c}$, (c) $0.8 M_{c}$, and (d) $1.0 M_{c}$.

and the grid numbers in the $x$ and $y$ directions are $N_{x} \times N_{y}=$ $384 \times 192,512 \times 256$ and $640 \times 320$, respectively. Figure 3 shows the statistical results of the mean and r.m.s streamwise velocities from the simulation using different grids, which achieve the self-similarity. It is observed that the independence results are achieved in different grid resolutions. The results from $512 \times 256$ and $640 \times 320$ are found to agree well.

The study of the grid independence on the statistics of the droplet-phase, including the mean streamwise velocity and r.m.s streamwise velocity of the droplets, is applied, and the results are illustrated in Figure 4. The profiles in different grid resolutions are found to achieve independence as the grid sets increase from $512 \times 256$ to $640 \times 320$. Based on the grid independence study for both gas-phase and droplet-phase, the computation grids are finally chose as $512 \times 256$ for the next-step investigation, considering the calculation costs.

\section{Results and Discussion}

3.1. Effects of Flow Compressibility on the Flow Features. Figure 5 shows the instantaneous distributions of Mach numbers, $M_{A}$, for the cases with $M_{c}$ increasing from 0.4 to 1.0 without droplet laden. The black lines refer to the dimensionless spanwise vorticity $\left(\Omega_{\mathrm{z}} /\left(\Delta U_{A} / \delta_{0}\right)=[-0.02,-0.005]\right.$ and indicate the shape of large-scale vortices in the shear layer. For the case with $M_{c}=0.4$, it is found that the supersonic flow loses the stability due to the shearing of the two streams, and the Kelvin-Helmholtz $(\mathrm{K}-\mathrm{H})$ instability. The flow processes of vortex rolling, shedding, pairing, merging, etc. in the shear layer occur, and the vortex structure is similar to that in the incompressible flow. With the increase of the flow compressibility, the streamwise location where the large-scale vortex begins to shed becomes far, and the shear layer flow is found to become difficult to lose its stability. From the results of the instantaneous flow fields, it is found that the vortex starts to shed at around the dimensionless streamwise distance $x / \delta_{0}=900$ for case $0.4 M_{c}$, while for case $1.0 \mathrm{M}_{c}$, the obvious shedding occurs until $x / \delta_{0}=$ 1300. At the same time, the increasing flow compressibility results in the obvious change of vortex shape from the regular near-circular structure $\left(M_{c}=0.4\right)$ to the strip-shaped flat structure stretched along the streamwise direction $\left(M_{c}=0.8\right.$ and 1.0) with a thinner shear layer thickness. From the spatial distributions of $M_{A}$, it is observed that the compression and expansion wave structures appear in the flow field with the increase of $M_{c}$, as shown in Figure 5(c) with $M_{c}=0.8$, and the shocklets around vortices occur. When $M_{c}$ increases to 1.0, although the vortex shape becomes flatter, the structure of shocklets becomes more conspicuous, and their intensity is also found to be enhanced. In particular, Figure 6 shows 


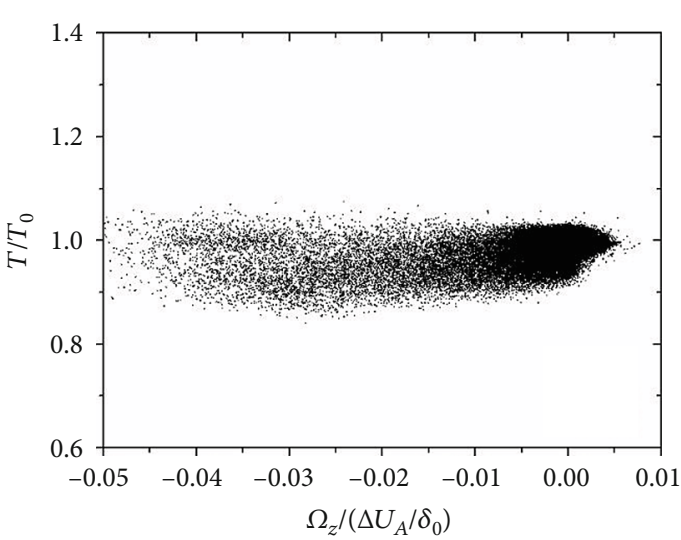

(a)

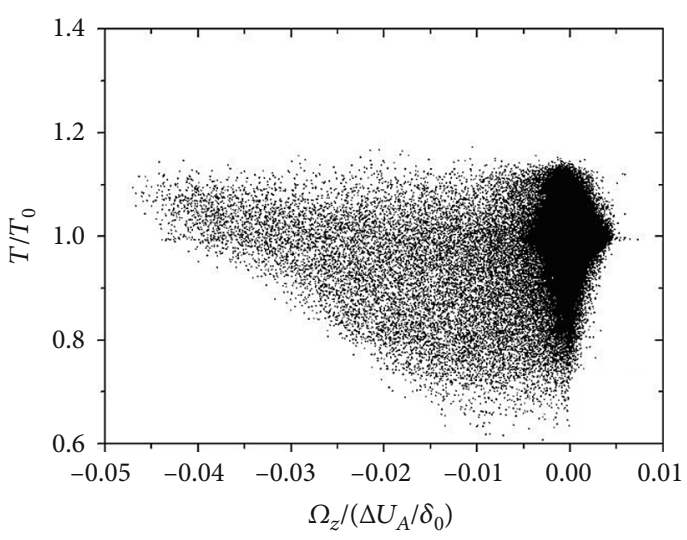

(c)

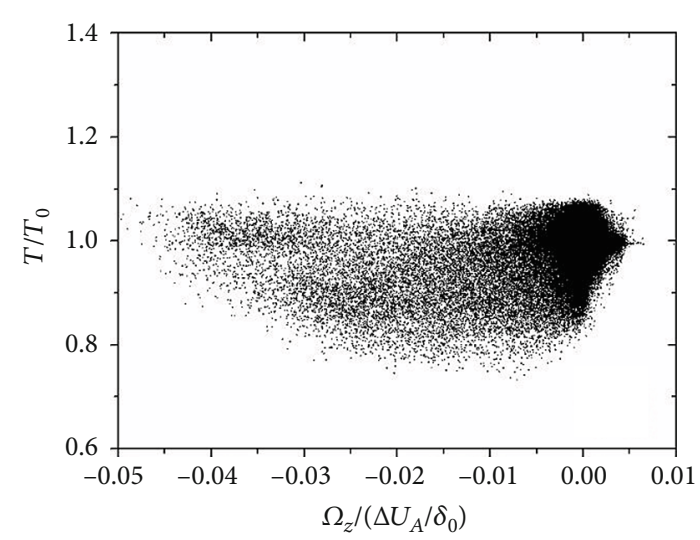

(b)

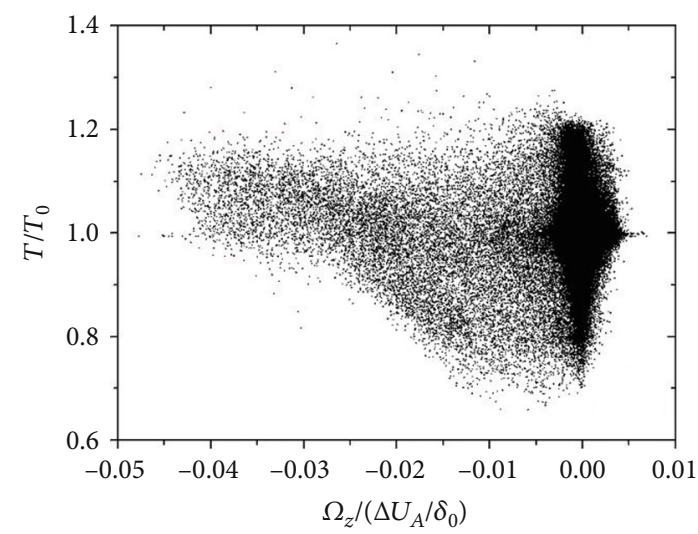

(d)

FIGURE 8: Scatter distributions of dimensionless temperature, $T / T_{0}$, in the coordinates of dimensionless spanwise vorticity, $\Omega_{\mathrm{z}} /\left(\Delta U_{\mathrm{A}} / \delta_{0}\right):($ a) $0.4 M_{c}$, (b) $0.6 M_{c}$, (c) $0.8 M_{c}$, and (d) $1.0 M_{c}$.

a shocklet for case $1.0 \mathrm{M}_{c}$, and the dashed line characterizes the shocklet with the solid lines indicating the streamlines. It can be found that the Mach number decreases as the airflow passes through the shocklet. The shocklet can be considered as a weak oblique shock wave. If the preshock Mach number is $M_{1}$, the flow direction is deflected after the oblique shock wave and the postshock Mach number is $M_{2}$. After the measurement of the shock wave angle of the shocklet in Figure 6 , the angle is $71.9^{\circ}$ and the pressure increase ratio $P_{2} / P_{1}$ equals 3.5 . For an oblique shock wave with angle $71.9^{\circ}$, the pressure increase ratio Posw ${ }_{2} / P_{\text {osw }}$ is 40.3 . Therefore, the intensity of the shocklet is much smaller than the intensity of the oblique shock wave with the same wave angle.

For the supersonic flows with high flow compressibility, the compression waves (shocklets) and expansion waves in the shear flow can lead to the obvious change of local pressure and temperature, which affects the evaporation of fuel droplets in the carrier flow. The distribution of temperature and pressure in the shear layers under the influence of flow compressibility will be analyzed below.

Figure 7 gives the instantaneous distributions of the dimensionless gas pressure, $P / P_{0}$, in the coordinates of dimensionless spanwise vorticity for the whole flow field. For case $0.4 \mathrm{M}_{\mathrm{c}}$, it is found that the maximum value of $P / P_{0}$ is around 1.0 and the minimum value is about 0.5 . These indicate that the compression waves are quite weak in the supersonic flow with low compressibility, and the pressure rise resulted from the compression process is unobvious. On the other hand, the expansion effect is relatively obvious. As the flow compressibility increases, such as cases $0.6 \mathrm{M}_{\mathrm{c}}$ and $0.8 \mathrm{M}_{c}$, the maximum pressure in the flow field increases significantly, and the values are found to be mainly located in the regions with low vorticity, namely, the high-strain vortexbraids between the vortices, which has relatively strong compression waves and even shocklets. It is also observed that the minimum pressure decreases as $M_{c}$ increases, indicating that the strength of the expansion waves also increases as the flow compressibility increases. For $M_{c}=1.0$, the compression and expansion in the supersonic shear layer flow are further intensified, and the pressure peaks in the high-strain, low-vorticity areas between the vortices is approximately doubled, compared with $P_{0}$, while the minimum value is found to have no significant change with that of case $0.8 M_{c}$. A high $M_{c}$ means that the acoustic time scale, $\delta / a$, is of the same order as the characteristic vortex stretching time scale $\delta / \Delta U$. This leads to the gas pressure to experience strong fluctuations during the turnover of the large-scale vortex, which is of the same order as the dynamic pressure and can also cause strong density fluctuations in the shear layer flow. When the free supersonic stream is entrained 


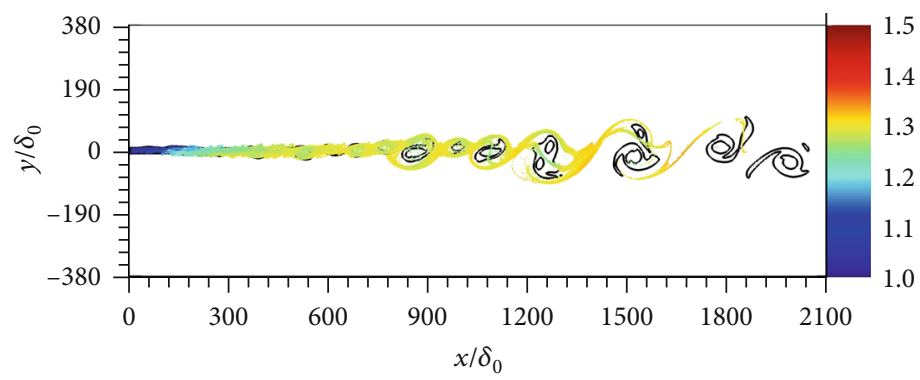

(a)

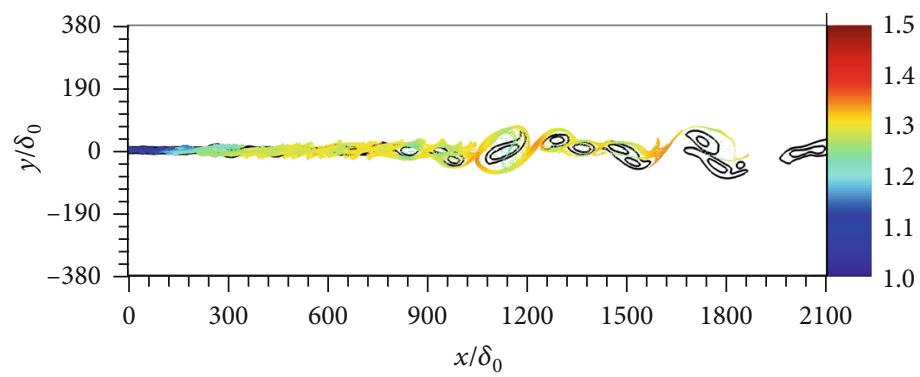

(b)

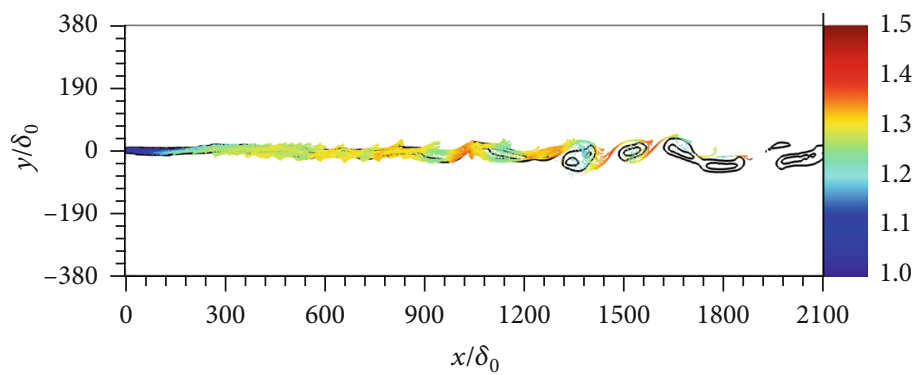

(c)

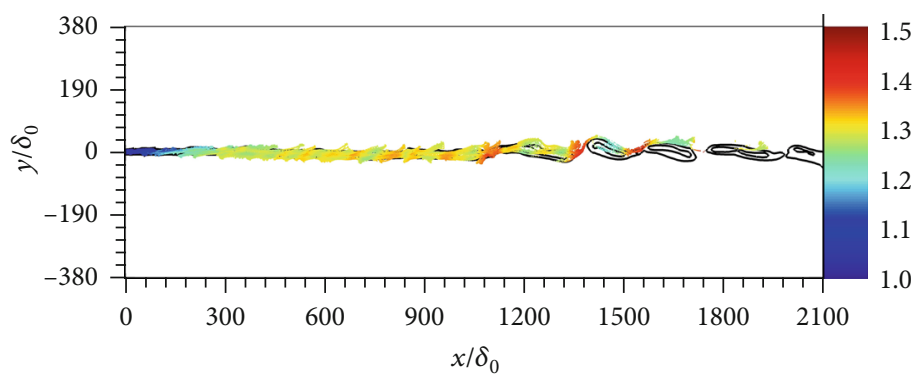

(d)

Figure 9: Instantaneous distributions of fuel droplets (color corresponding for the dimensionless droplet temperature $\left.T_{d} / T_{d, 0}\right)$ : (a) $0.4 M_{c}$, (b) $0.6 M_{c}$, (c) $0.8 M_{c}$, and (d) $1.0 M_{c}$. Here, the black lines refer to dimensionless spanwise vorticity $\left(\Omega_{\mathrm{z}} /\left(\Delta U_{\mathrm{A}} / \delta_{0}\right)=[-0.02,-0.005]\right.$.

into the shear layer, it can encounter the areas with the decrease of velocity, even subsonic areas. Sometimes, these conditions occur abruptly between vortices carrying supersonic and subsonic flows. These flow regions with strong Mach number fluctuations were called as eddy shocklet [28] or shocklet in the previous research, and the shocklets mainly appear in the flows with higher Mach numbers, as shown by the shocklets in the upper high-speed airflow of Figure 5.

Figure 8 shows the instantaneous distributions of the dimensionless gas temperature, $T / T_{0}$, in the coordinates of dimensionless spanwise vorticity for the whole flow field.
For the shear flow with low $M_{c}\left(M_{c}=0.4\right)$, it is found that the temperature decrease caused by the flow expansion effect is more pronounced than the temperature increase from the compression effect. As the flow compressibility increases, the temperature rise caused by the compression effect becomes obvious. In particular, for cases $0.8 M_{c}$ and $1.0 M_{c}$, it is clearly observed that the scatters with the temperature higher than $T_{0}$ are mainly concentrated in the regions with low-vorticity. When the free supersonic stream enters the shear layer, the high-speed compressibility causes the kinetic energy of the free stream to be converted into internal energy. Hence, the 


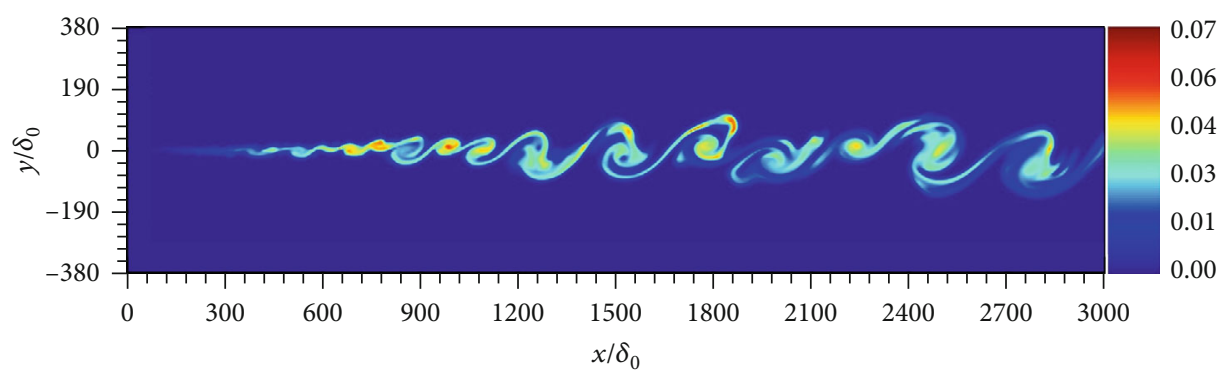

(a)

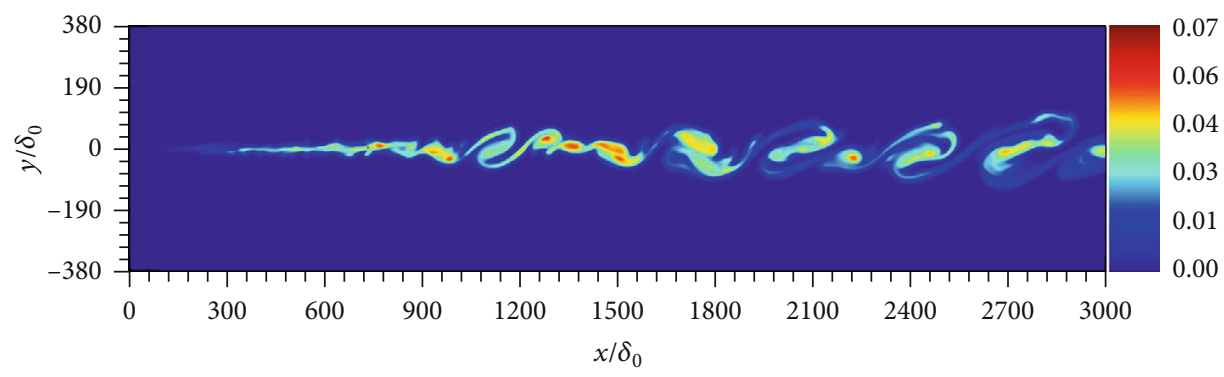

(b)

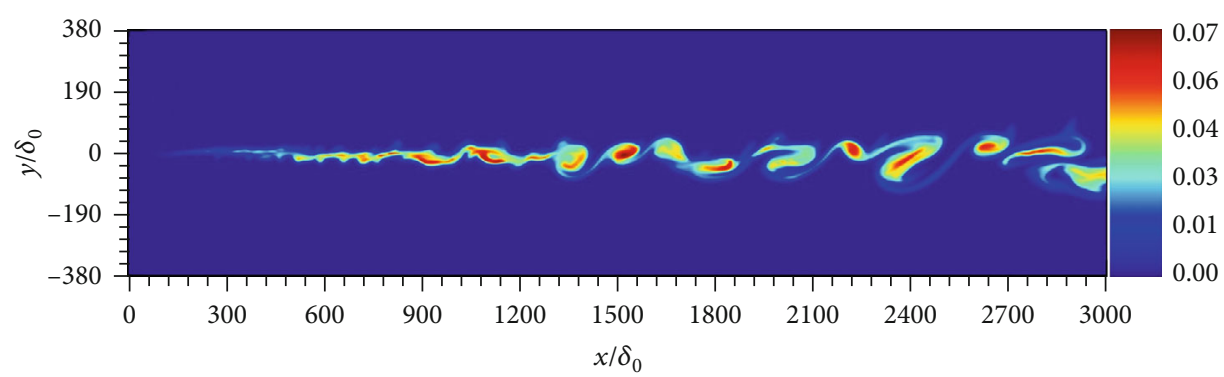

(c)

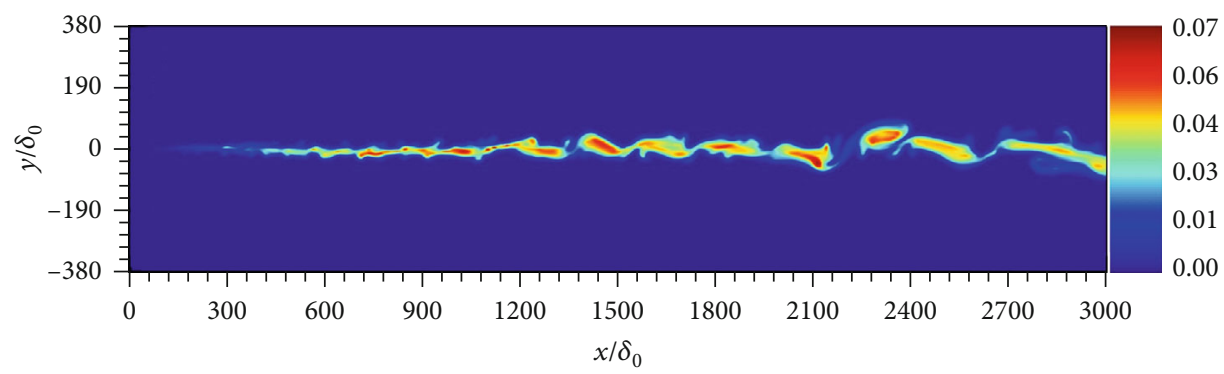

(d)

Figure 10: Instantaneous distributions of fuel vapor mass fraction $Y_{\mathrm{F}}$ : (a) $0.4 M_{c}$, (b) $0.6 M_{c}$, (c) $0.8 M_{c}$, and (d) $1.0 M_{c}$.

local gas temperature is increased by about 1.2 times than the temperature of the free stream, $T_{0}$, even if there is no chemical reaction. In addition, for the reactive flows with high compressibility, it is clear that a large part of the temperature rise in the flow field is due to the compressibility. The local hightemperature or low-temperature zone due to the flow compression or expansion effects will heat or cool the local fuel droplets, further affecting the evaporation, and these effects will be detailed in the following part.

3.2. Effects of Flow Compressibility on the Droplet Dispersion, Evaporation, and Fuel-Air Mixing. From the above analysis, the flow compressibility affects the structures and dynamics of vortices in the supersonic shear layer, and the expansion and compression waves in the flow field are found to influence the spatial distributions of the aerothermal parameters, such as pressure and temperature, of the carrier flow, which affects the dispersion and evaporation of the laden droplets.

Figure 9 gives the spatial distributions of fuel droplets in the supersonic shear flow. The selected vorticity indicates the vortex, and the dots represent the evaporating droplets with colors for the dimensionless droplet temperature $T_{d} / T_{d, 0}$. After the cold droplets disperse in the hot air stream, the heat 
is transferred from the surrounding gas to the droplets, associated with the increase of droplet temperature. For the shear flow with low compressibility $\left(M_{c}=0.4\right)$, the vortex structure is relatively regular, and the evaporating droplets tend to accumulate in the periphery of the vortices as well as the vortex-braids after the vortices are rolled up and shed. After the droplet temperature increases gradually from $T_{d, 0}$ to the saturation temperature, it remains almost unchanged in the downstream region. With the increase of flow compressibility, the temperature of the carrier flow in the vortexbraids is found to be increased, and it is observed that the temperature of droplets in the vortex-braids is much higher than that around the vortices. The increase in droplet temperature promotes evaporation. However, the flow expansion in the vortex cores causes the local gas temperature to decrease, associated with the decrease of the droplet temperature, which inhibits the evaporation. The increase of flow compressibility results in the compression of the vortex structure along the transverse direction, and hence the distribution of droplets entrained by the vortex is found to be narrow.

Figure 10 shows the instantaneous distribution of the fuel vapor mass fraction $Y_{F}$ in the supersonic shear layer. The spatial distribution of the fuel vapors depends on the injection position of the droplets as well as the dispersion characteristics of the evaporative droplets. The droplets are initially injected in the central region of the shear layer inlet before the vortex starts to shed, and the fuel vapors are enriched in the central region. For case $0.4 M_{c}$, the droplets segregate in the central region of the shear layer before the vortex shedding, and the fuel vapors are enriched locally. After the vortex is shed, the vortex entrains vapors in the vortex cores. The turbulent motion of the vortex entrains the droplets, resulting in the preferential accumulation of droplets in the periphery of the vortex as well as the vortex-braid, and it is found that a large number of fuel vapors exists around the vortex. With the increase of flow compressibility, the vortex is compressed along the transverse direction and the structure becomes narrow, and the dispersion region of droplets is also narrowed, resulting in the concentrated distribution of fuel vapors. For case $1.0 M_{c}$, it is observed that the fuel vapors are mainly concentrated inside the vortices. There are two main reasons attributed to this phenomenon. On one hand, the flow becomes difficult to lose the stability with the increase of flow compressibility, and the location for the rolling-up and shedding of vortices is found to move downstream. The droplets have evaporated to form many fuel vapors before the vortex shedding. Since the droplets segregate in the central region of the shear layer, the fuel vapors are concentrated in the shear layer center. As the vortex is shed, most of the fuel vapors accumulate inside the vortices. On the other hand, the flow compressibility affects the dynamics of vortices, the droplets entrained by the vortices distribute in the central region of the shear layer, and hence the fuel vapors are concentrated in the shear layer center.

In order to analyze the mixing degree of fuel vapors with air, the spatial mixing deficiency (SMD) [29] is used, which

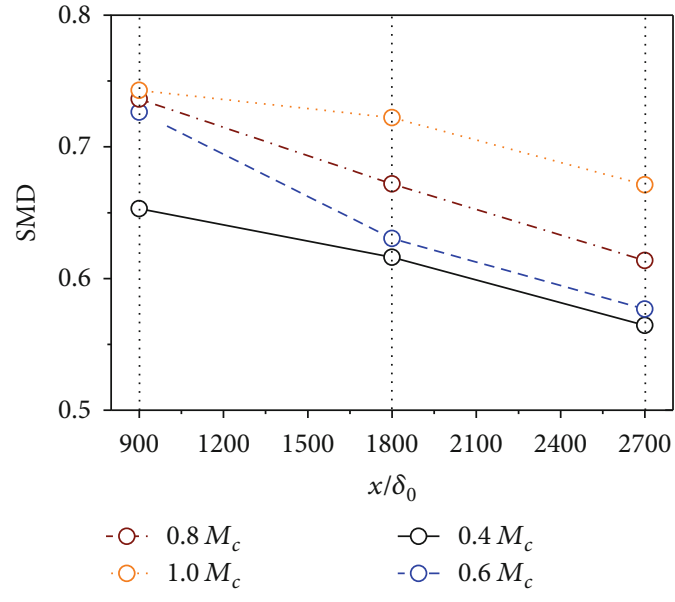

FIGURE 11: The distribution of SMD along the streamwise direction.

measures the ununiform distribution of chemical components. The expression is as follows,

$$
\mathrm{SMD}=\frac{\operatorname{RMS}_{\text {plane }}\left(\bar{Y}_{i}\right)}{\operatorname{Avg}_{\text {plane }}\left(\bar{Y}_{i}\right)}
$$

where RMS is the root mean square of the time-averaged species at a certain plane, $\bar{Y}_{i}$,

$$
\operatorname{RMS}_{\text {plane }}\left(\bar{Y}_{i}\right)=\sqrt{\frac{1}{m_{\text {plane }}-1} \sum_{i=1}^{m_{\text {plane }}}\left(\bar{Y}_{i}-\operatorname{Avg}_{\text {plane }}\left(\bar{Y}_{i}\right)\right)^{2}}
$$

and Avg is the mean value of $\bar{Y}_{i}$,

$$
\operatorname{Avg}_{\text {plane }}\left(\bar{Y}_{i}\right)=\frac{1}{m_{\text {plane }}} \sum_{i=1}^{m_{\text {plane }}}\left(\bar{Y}_{i}\right)
$$

$m_{\text {plane }}$ is the number of grids on this plane. As SMD equals to 0 , it represents a complete mixing at this plane. For the mixing process in the supersonic shear layer, three transverse planes along the streamwise direction are selected, and $x / \delta_{0}$ is taken as 900,1800 , and 2700 for the evaporating, the completion of evaporation, and the downstream regions, respectively. Figure 11 shows the distributions of SMD at different streamwise locations. It is found that the mixing is gradually completed with the development of the shear layer, and the SMD decreases. For the same streamwise location, the SMD increases with the increasing $M_{c}$, which indicates that the mixing is insufficient and demonstrates that the increase of flow compressibility inhibits the mixing of the chemical components.

\section{Conclusions}

In the present study, we present a systematic investigation for the influence of high-speed flow compressibility on the dispersion and evaporation of droplets, and two-phase mixing 
in supersonic shear layers. The fuel droplets interact with the turbulent shear flows through the two-way coupling model, and the Eulerian-Lagrangian point source approach is adopted. The convective Mach numbers of the carrier flows increase from 0.4 to 1.0 .

The overall features of the aerothermal field of the supersonics shear layer are first analyzed, and the effects of the flow compressibility are discussed. The compression and the dilatation in the compressible flow are found to be spatially intermittent, which is attributed to the high-speed flow compressibility, and they result in the switching between thermal and kinetic energies during the turnover of the large-scale vortices. The flow compressibility influences the carrier flow, which affects the dispersion, evaporation, and mixing of droplets. On one hand, the shear vortices are compressed along the transverse direction, and their sizes reduce as the flow compressibility increases. Therefore, the dispersion range of the fuel droplets transported by the local vortices becomes narrow, associated with the reduced distribution of fuel vapors from evaporation. On the other hand, it is found that the intermittent spatial distributions of the expansion zone with low temperature and the compression zone with high temperature occur due to the high flow compressibility and affect the heat and mass transfers from the carrier gas to the droplets. The strengthen of the flow compressibility reduces the mixing degree of fuel droplets. Generally, the increasing convective Mach number is found to suppress the dispersion, evaporation, and mixing. However, the increasing temperature of the carrier gas due to the flow compressibility is expected to contribute to the ignition and combustion, and the effects on combustion are worth further investigation.

\section{Data Availability}

Data available on request.

\section{Conflicts of Interest}

The authors declare that they have no conflicts of interest.

\section{Acknowledgments}

This work is partially supported by NSFC under the Grant No. 51676111 and 51806179, NSFC-NAF under the Grant No. U1730104, the project funded by China Postdoctoral Science Foundation, the Fundamental Research Funds for the Central Universities, and Basic Research Plan of Natural Science in Shaanxi Province.

\section{References}

[1] W. Ao, Z. Chen, P. Liu, S. Shang, K. Ma, and B. Fu, "Mixing enhancement in a subsonic-supersonic shear layer with a cavity splitter plate," Aerospace Science and Technology, vol. 102, article 105847, 2020.

[2] K. Ma, J. Li, Q. Li, and Y. Liu, "Experimental study on evolution characteristics of plane subsonic-supersonic shear layer," Aerospace Science and Technology, vol. 100, p. 105791, 2020.

[3] G. Xiang, Z. Ren, S. Kim, and B. Wang, "Numerical analysis on the disintegration of gas-liquid interface in two-phase shear- layer flows," Aerospace Science and Technology, vol. 98, p. $105710,2020$.

[4] P. Guo, Z. Gao, Z. Wu, H. Liu, C. Jiang, and C. Lee, "Investigations on the accurate prediction of supersonic shear layers for detached eddy simulation," Aerospace Science and Technology, vol. 89, pp. 46-57, 2019.

[5] G. L. Brown and A. Roshko, "On density effects and large structure in turbulent mixing layers," Journal of Fluid Mechanics, vol. 64, no. 4, pp. 775-816, 1974.

[6] D. Papamoschou and A. Roshko, "The compressible turbulent shear layer: an experimental study," Journal of Fluid Mechanics, vol. 197, pp. 453-477, 1988.

[7] N. Chinzei, G. Masuya, T. Komuro, A. Murakami, and K. Kudou, "Spreading of two-stream supersonic turbulent mixing layers," The Physics of Fluids, vol. 29, no. 5, pp. 13451347, 1986.

[8] N. T. Clemens and M. G. Mungal, "Large-scale structure and entrainment in the supersonic mixing layer," Journal of Fluid Mechanics, vol. 284, pp. 171-216, 1995.

[9] A. Kourta and R. Sauvage, "Computation of supersonic mixing layers," Physics of Fluids, vol. 14, no. 11, pp. 3790-3797, 2002.

[10] A. W. Vreman, N. D. Sandham, and K. H. Luo, "Compressible mixing layer growth rate and turbulence characteristics," Journal of Fluid Mechanics, vol. 320, pp. 235-258, 1996.

[11] B. Wang, W. Wei, Y. Zhang, H. Zhang, and S. Xue, "Passive scalar mixing in $\mathrm{Mc}<1$ planar shear layer flows," Computers \& Fluids, vol. 123, pp. 32-43, 2015.

[12] G. S. Elliott and M. Samimy, "Compressibility effects in free shear layers," Physics of Fluids A: Fluid Dynamics, vol. 2, no. 7, pp. 1231-1240, 1990.

[13] M. G. Olsen and J. C. Dutton, "Planar velocity measurements in a weakly compressible mixing layer," Journal of Fluid Mechanics, vol. 486, pp. 51-77, 2003.

[14] C. Pantano and S. Sarkar, "A study of compressibility effects in the high-speed turbulent shear layer using direct simulation," Journal of Fluid Mechanics, vol. 451, pp. 329-371, 2002.

[15] A. Atoufi, M. Fathali, and B. Lessani, "Compressibility effects and turbulent kinetic energy exchange in temporal mixing layers," Journal of Turbulence, vol. 16, no. 7, pp. 676-703, 2015.

[16] A. Burcat and B. Ruscic, "Third millenium ideal gas and condensed phase thermochemical database for combustion (with update from active thermochemical tables)," in Argonne National Lab, (ANL), Argonne, IL (USA), 2005.

[17] B. E. Poling, J. M. Prausniz, and J. P. O'Connell, The Properties of Gases and Liquids, McGraw-Hill, New York, 2001, Chaps.

[18] Z. Ren, B. Wang, Q. Xie, and D. Wang, "Thermal auto-ignition in high-speed droplet-laden mixing layers," Fuel, vol. 191, pp. 176-189, 2017.

[19] R. S. Miller, K. Harstad, and J. Bellan, "Evaluation of equilibrium and non-equilibrium evaporation models for manydroplet gas-liquid flow simulations," International Journal of Multiphase Flow, vol. 24, no. 6, pp. 1025-1055, 1998.

[20] Z. Ren, B. Wang, and L. Zheng, "Numerical analysis on interactions of vortex, shock wave, and exothermal reaction in a supersonic planar shear layer laden with droplets," Physics of Fluids, vol. 30, no. 3, article 036101, 2018.

[21] X. Y. Hu, Q. Wang, and N. A. Adams, "An adaptive centralupwind weighted essentially non-oscillatory scheme," Journal of Computational Physics, vol. 229, no. 23, pp. 8952-8965, 2010. 
[22] Y. Zhang, B. Wang, H. Zhang, and S. Xue, "Mixing enhancement of compressible planar mixing layer impinged by oblique shock waves," Journal of Propulsion and Power, vol. 31, no. 1, pp. 156-169, 2014.

[23] T. J. Poinsot and S. K. Lelef, "Boundary conditions for direct simulations of compressible viscous flows," Journal of Computational Physics, vol. 101, no. 1, pp. 104-129, 1992.

[24] M. Mikami, Y. Mizuta, Y. Tsuchida, and N. Kojima, "Flame structure and stabilization of lean-premixed sprays in a counterflow with low-volatility fuel," Proceedings of the Combustion Institute, vol. 32, no. 2, pp. 2223-2230, 2009.

[25] A. L. Sánchez, J. Urzay, and A. Liñán, “The role of separation of scales in the description of spray combustion," Proceedings of the Combustion Institute, vol. 35, no. 2, pp. 1549-1577, 2015.

[26] S. G. Goebel, J. C. Dutton, H. Krier, and J. P. Renie, "Mean and turbulent velocity measurements of supersonic mixing layers," Mineralium Deposita, vol. 29, no. 1, pp. 263-272, 1990.

[27] G. Xu, M. Ikegami, S. Honma et al., "Inverse influence of initial diameter on droplet burning rate in cold and hot ambiences: a thermal action of flame in balance with heat loss," International Journal of Heat and Mass Transfer, vol. 46, no. 7, pp. 1155-1169, 2003.

[28] S. Lee, S. K. Lele, and P. Moin, "Eddy shocklets in decaying compressible turbulence," Physics of Fluids A: Fluid Dynamics, vol. 3, no. 4, pp. 657-664, 1991.

[29] C. Priere, L. Y. M. Gicquel, P. Kaufmann, W. Krebs, and T. Poinsot, "Large eddy simulation predictions of mixing enhancement for jets in cross-flows," Journal of Turbulence, vol. 5, no. 5, pp. 1-24, 2004. 